\title{
Generation of high-resolution kagome lattice structures using extreme ultraviolet interference lithography
}

\author{
Li Wang, ${ }^{1, a)}$ Bernd Terhalle, ${ }^{1}$ Vitaliy A. Guzenko, ${ }^{1}$ Alan Farhan, ${ }^{1}$ Mohamad Hojeij, ${ }^{1}$ \\ and Yasin Ekinci ${ }^{1,2}$ \\ ${ }^{1}$ Laboratory for Micro- and Nanotechnology, Paul Scherrer Institute, CH-5232 Villigen PSI, Switzerland \\ ${ }^{2}$ Laboratory of Metal Physics and Technology, Department of Materials, ETH Zürich, CH-8093 Zürich, \\ Switzerland
}

(Received 5 July 2012; accepted 14 August 2012; published online 27 August 2012)

\begin{abstract}
High-resolution kagome lattice structures with feature sizes down to the sub-50 nm regime are fabricated using diffraction-based extreme ultraviolet interference lithography. The resulting interference pattern of multiple beams is sensitive to the relative phase of the interfering beams. The precise control of their phases is achieved by precise positioning of transmission diffraction gratings on a mask using a high-end electron beam lithography tool. The presented method may find applications in providing high-resolution and large-area kagome lattice structures for studies on frustrated magnetic systems, photonic crystals, and plasmonics. (C) 2012 American Institute of Physics. [http://dx.doi.org/10.1063/1.4748758]
\end{abstract}

A kagome lattice, a well-known traditional pattern for centuries, is a two-dimensional (2D) pattern composed of corner-shared triangles whose lattice points have four nearest neighbors each. ${ }^{1}$ Especially in condensed matter physics, such structures have attracted special attention as they provide, e.g., one of the most geometrically frustrated magnetic system. ${ }^{2}$ The interesting feature of a 2D kagome lattice is that it has dispersionless flatbands in its band structure. It has been theoretically shown that ferromagnetism appears in such a lattice when the flatbands are half filled. ${ }^{3}$ Even though the kagome lattice has been the subject of intensive theoretical studies, further developments are rather dependent on the experimental realization, which is a technologically challenging task. Recently, a selective area metallo-organic vapor phase epitaxy method has been utilized to realize semiconductor artificial kagome lattice. ${ }^{4}$ However, design of kagome lattice structure with a smaller period is not feasible with their setup. In current research, one of the key requirements in the studies of frustrated physical systems is to achieve smaller kagome structures, in order to bring the patterned nanomagnet islands approach the superparamagnetic limit, which is required for achieving a dynamic regime in an artificial spin ice system, as an example. ${ }^{5}$ However, thus far, such required small size islands arranged in a kagome lattice have not even been realized using electron beam lithography (EBL). ${ }^{6}$ Nonetheless, Gaussian-shaped EBL has low throughput because of the serial writing nature of this technique. Additional challenges are precise control of the shape of the individual islands and the distance between them. These arise from the discrete addressing grid of the beam deflection system, which is usually a drawback for exposure of the arbitrary shaped structures. Recently, Boguslawski et al. have reported on the development and generation of a non-diffracting kagome beam at a wavelength of $532 \mathrm{~nm}$ by using phase modulators and have demonstrated the generation of $2 \mathrm{D}$ kagome lattices with a

\footnotetext{
${ }^{\text {a) }}$ Author to whom correspondence should be addressed. Electronic mail: li.wang@psi.ch.
}

holographic matter structuring technique. ${ }^{7}$ Recently, they introduced a new concept for the generation of other kinds of non-diffracting beams and demonstrated complex structures with nonlinear photorefractive medium. ${ }^{8}$ However, the realization of kagome lattice structures of the nanometer scale on solid samples using photon-based lithography method for aforementioned condensed matter research as well as nanophotonics is still missing.

Extreme ultraviolet (EUV) lithography is currently considered to be the next-generation technique for high-volume semiconductor chip production with feature sizes of $22 \mathrm{~nm}$ and below. ${ }^{9}$ In parallel with development of projection optical systems for EUV lithography, EUV interference lithography (EUV-IL) gained special attention in both academic and industrial research as a powerful tool capable of providing high resolution, high throughput, and large-area patterning. ${ }^{10}$ In the most common EUV-IL experimental setup, two transmission gratings on a membrane mask are illuminated with an EUV beam. The gratings diffract the beam into multiple diffraction orders, which form at a certain distance with an interference pattern with the period equal to $1 /(2 m)$ of the period of the gratings on mask, where $m$ is the diffraction order. In order to obtain more versatile patterns, multiple beams should be used and mixed together. However, when the number of interfering beams exceeds three, the intensity pattern depends strongly on the relative phases of the beams. This opens up the potential to generate complex periodic as well as quasi-crystal like structures using EUV-IL. ${ }^{11,12}$ In our recent numerical simulations, we reported that hexagonal arrays of dots and honeycomb structures as well as kagome lattice could be obtained by interference of six beams with well-controlled relative phases. ${ }^{11}$ In this paper, we report the experimental realization of kagome lattice structures using EUV-IL with e-beam written gratings with precise placement and orientation.

Since the kagome lattice possesses a six-fold symmetry, it is self-evident to start with the simulation of the interference of six coherent plane waves with individual wave vectors $k_{n}(\mathrm{n}=1,2 \ldots 6)$. These beams can be created by circular 
arrangement of six transmission diffraction gratings with period $P$ such that the corresponding first diffraction orders overlap in the substrate plane and form a Fourier space cone with an opening angle of $2 \theta$, as depicted in Fig. 1(a). The opening angle of the Fourier space cone formed by the six beams is determined by the first-order diffraction angle

$$
\theta=\arcsin (\lambda / P) .
$$

The transverse wave vector component of the beams is defined by the opening angle of the cone and expressed as

$$
k_{t, n}=\left|k_{n}\right| \sin (\theta)=(2 \pi / \lambda) \times(\lambda / P)=2 \pi / P .
$$

And the longitudinal wave vector component, which is pointing in $z$ direction in Fig. 1, is

$$
k_{l, n}=\sqrt{\left|k_{n}\right|^{2}-k_{t, n}^{2}} .
$$

It has been demonstrated in the visible as well as in the EUV wavelength range that, in order to obtain non-diffracting kagome beam, the relative phase shift of all the beams should be $4 \pi .^{7,11}$ In the interference scheme based on transmission diffraction gratings, phase shift can be achieved by introducing a radial shift $\Delta_{r, n}$ of one or more gratings, i.e., in the direction perpendicular to the grating lines. ${ }^{11}$ The path difference resulting from the shift of a single grating can be written as

$$
\phi_{n}=\Delta_{r, n} \sin (\theta)=\Delta_{r, n} \lambda / P
$$

Apparently, according to Eq. (4), a desired relative phase shift of $4 \pi$ can be achieved by a total shift of $2 P$, e.g., by shifting the corresponding gratings by $n \times 2 P / 6$ with $n=1$ to 6, as depicted in Fig. 1(a). Finally, the recorded pattern can be calculated using the field distribution of the six interfering beams by

$$
I_{\text {tot }}=\sum_{n=1}^{6} \exp \left(i\left(k_{x, n}\left(x-\Delta_{x, n}\right)+k_{y, n}\left(y-\Delta_{y, n}\right)+k_{l, n} z\right)\right) \text {, }
$$

where $\Delta_{x, n}=\Delta_{r, n} k_{x, n} / k_{t, n}$ and $\Delta_{y, n}=\Delta_{r, n} k_{y, n} / k_{t, n}$.

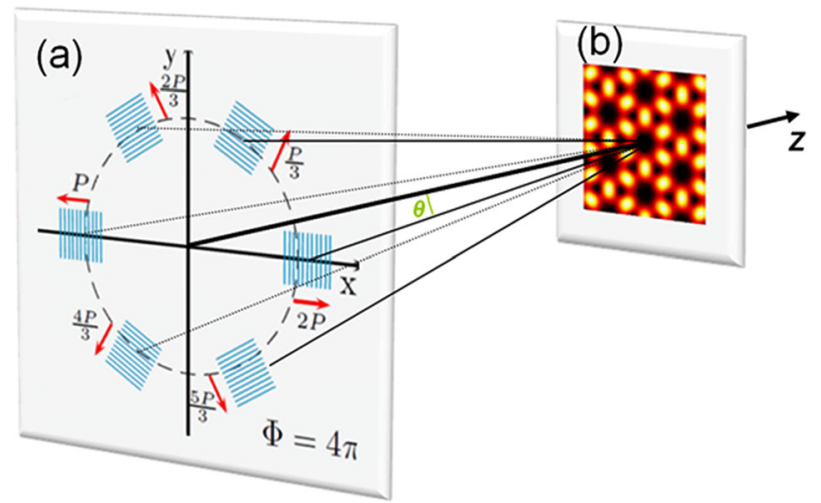

FIG. 1. (a) Schematic illustration of the mask design of the phase engineered multiple transmission diffraction gratings mask for EUV-IL. The red arrows indicate the radial shift direction of the individual gratings with respect to the original grating positions in order to get an aerial image of kagome lattice. (b) Numerical simulation of the intensity distribution of the aerial image generated with this mask.
Fig. 1(b) illustrates the simulated intensity distribution of the interference pattern $I(x, y)=\left|I_{t o t}\right|^{2}$ of the six phaseengineered beams with a total phase shift of $4 \pi$. The resulting intensity distribution is clearly a kagome lattice structure.

For the experimental realization, a mask has been fabricated on a 100 -nm-thick $\mathrm{Si}_{3} \mathrm{~N}_{4}$ membrane, consisting of six identical transmission diffraction gratings with radial displacement from a circle as illustrated in Fig. 1(a). First, the electroplating base was deposited on the membranes by thermal evaporation of $\mathrm{Cr}(2 \mathrm{~nm}) / \mathrm{Au}(5 \mathrm{~nm}) / \mathrm{Cr}(2 \mathrm{~nm})$ multilayer. Then, the samples were spin-coated with a $220 \mathrm{~nm}$ thick layer of negative tone resist hydrogen silsesquioxane (HSQ) (FOX 16, Dow Corning). The grating patterns were exposed with a Vistec EBPG5000Plus EBL tool operated at $100 \mathrm{keV}$ electron energy. Special attention has been paid to the preparation of the exposure data, in particular, in order to minimize the effect of the discrete addressing grid, a very high resolution of the exposure pattern had to be chosen. This way, the rounding error and thus the possible jitter of the grating period as well as the line width dependence on the grating orientation were minimized. Additionally, all six gratings were fitted into one writing field, which eliminated stitching errors due to the stage movements and increased the placement precision of individual gratings in respect to each other. Following the exposure, samples were developed in a $1 \%$ aqueous solution of $\mathrm{NaOH}$. Obtained arrays of HSQ lines served as a hard mask for the following reactive ion etching process based on $\mathrm{Cl}_{2} / \mathrm{CO}_{2}$ plasma. Thereby, the top $\mathrm{Cr}$ layer of the electroplating base was removed in order to facilitate the subsequent $\mathrm{Au}$ electroplating step. Thus, grating bars with duty cycle of $\sim 0.5$ and a thickness of approximately $100 \mathrm{~nm}$ as well as closed Au film on remaining regions of the membrane were formed. ${ }^{13}$ This thickness of $\mathrm{Au}$ thin film is sufficient to absorb $99.6 \%$ of incident EUV light, and our calculations show that these Au gratings provide $12 \%$ first order diffraction efficiency with respect to the 0th order in the EUV spectral range we used. Taking into account the limited mechanical stability of the membranes, parameters of the electroplating process were optimized in order to obtain lowstress $\mathrm{Au}$ thin films. In the end, HSQ plating templates were removed in buffered oxide etcher (BOE). Using this approach for EUV-IL transmission mask fabrication allowed us to simplify remarkably the process flow and to minimize the potential phase errors characteristic for the multiple-step mask fabrication processes, which require additional overlay exposure of the central photon stop layer. ${ }^{14}$

Gratings with different periods were fabricated to form kagome lattices with various sizes as well as periods. The quality of the gratings was characterized by polarization contrast optical microscopy as well as scanning electron microscopy (SEM). The different intensities in optical dark field images are due to the orientation of the gratings with respect to the polarizer. As shown in Fig. 2 for the period of $300 \mathrm{~nm}$, homogeneous $\mathrm{Au}$ gratings, arranged in hexagonal shape, with smooth line edges and filling factor of $\sim 0.5$ for all six gratings were fabricated on $\mathrm{Si}_{3} \mathrm{~N}_{4}$ membrane with designed phase relations.

The EUV exposures were performed at the XIL-II beamline at the Swiss Light Source (SLS) with spatially coherent EUV light of $13.5 \mathrm{~nm}$ wavelength with a bandwidth 


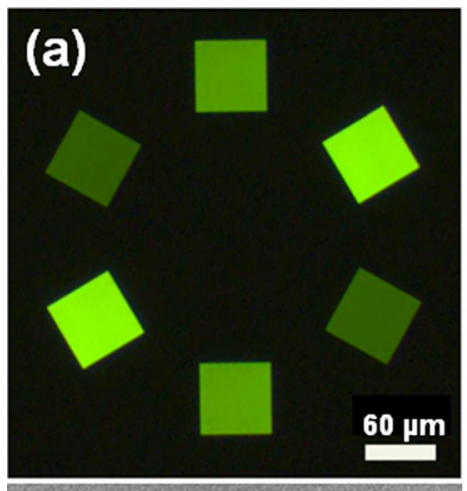

(b) $60 \mathrm{~mm}$
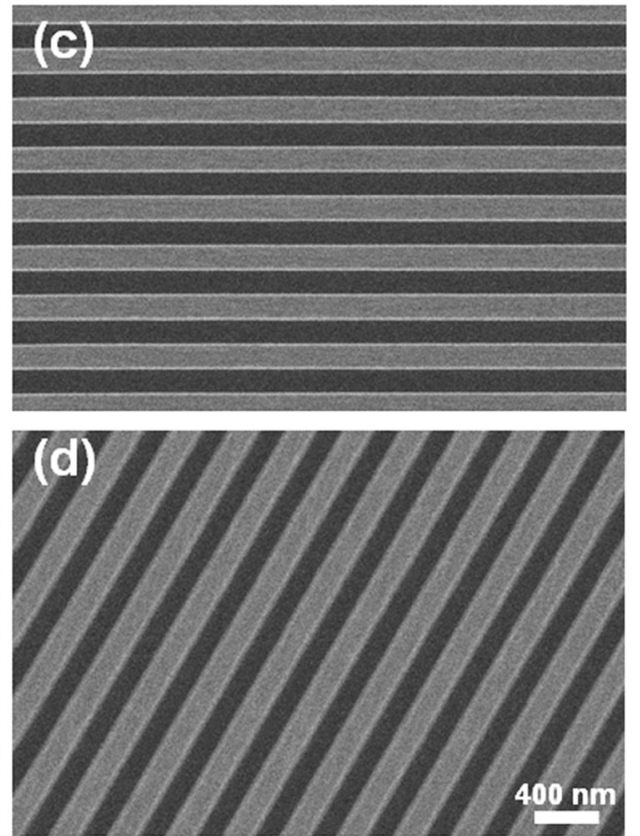

FIG. 2. (a) Polarization contrast optical microscopy and (b) scanning electron microscopy images of six grating mask with period of $300 \mathrm{~nm}$ on a 100 -nm-thick $\mathrm{Si}_{3} \mathrm{~N}_{4}$ membrane. (c) and (d) are the magnified SEM images showing the $100 \mathrm{~nm}$ thick Au gratings with $\sim 0.5$ filling factor for horizontal and $60^{\circ}$ tilted gratings in (b), respectively. of $\Delta \lambda / \lambda=4 \%$. The undulator generated EUV radiation has been used without spectral filtering, except for elimination of higher-order harmonics, which is achieved by a sufficiently high incident angle of reflection from mirrors in the beamline. Before the masks were mounted to the beamline, the careful alignment of the beam had to be performed: the beam was centered and set normal to the mask. To record the interference patterns from these phase engineered multiple gratings, HSQ resist (FOX XR1541) was spincoated on Si wafers at a speed of $5000 \mathrm{rpm}$ for $45 \mathrm{~s}$, resulting in a thickness of 35-nm-thick film. The gap between mask and sample was set to designed values and the exposure dose was varied between 10 and $100 \mathrm{~mJ} / \mathrm{cm}^{2}$. After the exposure, the samples were developed in a $25 \%$ aqueous solution of tetramethylammonium hydroxide (TMAH) for $60 \mathrm{~s}$. The results are summarized in Fig. 3: SEM images of the low

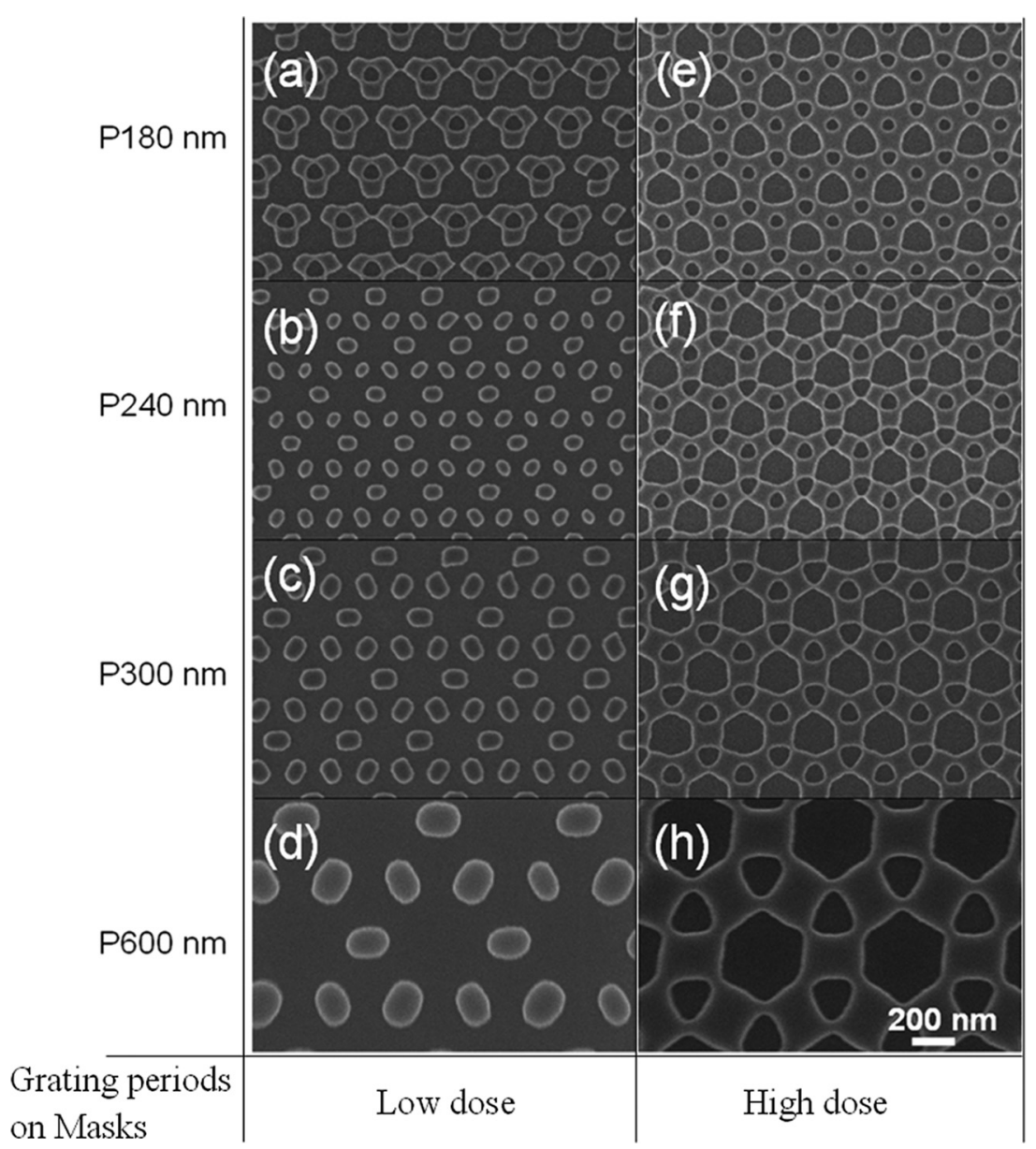

FIG. 3. Experimental realization of six-beam interference with $4 \pi$ phase shift to generate kagome lattice structures. SEM images show kagome lattice with various sizes and periods recorded by interference from gratings having different periods, from $180 \mathrm{~nm}((\mathrm{a}),(\mathrm{e}))$ to $600 \mathrm{~nm}((\mathrm{~d})$, (h)). Left ((a)-(d)) and right column ((e)-(h)) SEM images refer to low dose and high dose EUV-IL exposures, respectively. 

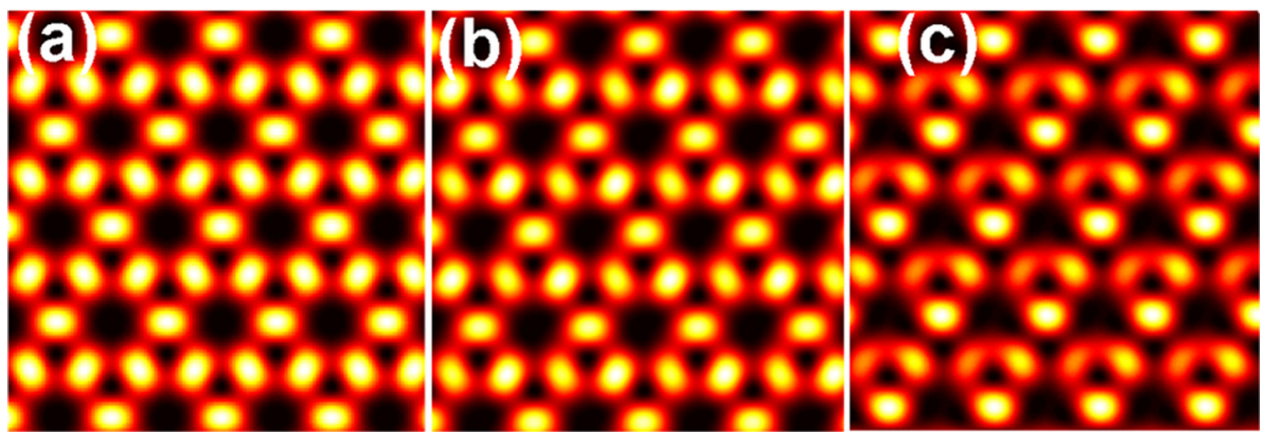

FIG. 4. Simulated intensity pattern of an ideal six-beam interference with $4 \pi$ phase shift using gratings having period of $180 \mathrm{~nm}(\mathrm{a})$, and in the case when one of the gratings is shifted by $5 \mathrm{~nm}$ (b) and $10 \mathrm{~nm}(\mathrm{c})$, respectively.

dose exposure (Figs. 3(a)-3(d)) indicate the formation of resist patterns, which are in very good agreement with the simulated intensity maps in Fig. 1(b) except for recorded kagome structures with period of $180 \mathrm{~nm}$ (Fig. 3(a)), which showed partially distorted arrangement compared to the ideal pattern. Nevertheless, kagome lattice structures with various island sizes (from 40 to $200 \mathrm{~nm}$ ) and periods (from 180 to $600 \mathrm{~nm}$ ) were recorded on Si wafers using six-beam interference from phase engineered transmission diffraction gratings with different periods (from 180 to $600 \mathrm{~nm}$ ) on masks, respectively (see Fig. 3). At high exposure dose, the shape of the recorded kagome lattice structures results in merging of the islands and formation of the hexagonal shaped holes surrounded by six triangular shaped ones (Figs. 3(e)-3(h)).

In the multiple beam interference, amplitude and phase errors of individual beams lead to variations in the final interference patterns. ${ }^{12}$ In the present experimental configuration, amplitude errors may result from unequal EUV beam intensity distribution on gratings and grating efficiency, which might be caused mainly by variation of duty cycles of the written gratings. Phase errors arise from displacement of gratings or misalignment between the mask and EUV beam. Here, the partial distortion of recorded kagome structures in Fig. 3(a) could be well confirmed with numerical simulations. Fig. 4 shows the calculated intensity distributions for different cases. Fig. 4(a) shows the resulting pattern for the ideal case of the six-beam interference with gratings with period of $180 \mathrm{~nm}$. Figs. 4(b) and 4(c) represent the cases where the relative position of one of the six gratings has been shifted by $5 \mathrm{~nm}$ and $10 \mathrm{~nm}$ along the radial axis, respectively. As seen in Fig. 4(c)), a significant distortion of intensity distribution is observed, which is in good agreement with the SEM image in Fig. 3(a). The misplacement of the grating by $10 \mathrm{~nm}$ corresponds to about $P / 20$. In case of a shift of one of the gratings by only $5 \mathrm{~nm}$ resulted in a kagome lattice with reasonable fidelity as shown in Fig. 4(b). This also gives a way to roughly estimate the tolerance of phase errors induced by imprecise placement of gratings with various periodicities on masks, which may be introduced by stitching errors or noise during e-beam writing of the gratings, for example. Although we carried out the simulations for the misplacement of a single grating, in reality, this probably occur randomly or systematically for all individual gratings. In addition, we cannot rule out other factors that are limiting the resolution such as the minor phase errors induced to the mask gratings during EBL or other mask fabrication steps and the alignment of the EUV beam to mask and substrate. Nevertheless, we can conclude that for the fabrication of the kagome lattice with good fidelity, the precision of the gratings must be better than $P / 20$. For smaller periods, such a high precision in positioning over several hundred microns is certainly challenging, which can be overcome with better calibration of the e-beam optics and new writing strategies.

In conclusion, we have demonstrated the fabrication of kagome lattice structures using diffraction-based EUV-IL of six beams with well-controlled relative phase relations. The phase shifts of the beams were realized by precise placement of transmission diffraction gratings on a mask membrane using EBL and careful alignment of the mask and the primary beam. Efficient diffraction grating masks were fabricated using a simplified two-step process. We have demonstrated kagome lattices with feature sizes down to $40 \mathrm{~nm}$ and periods down to $180 \mathrm{~nm}$. We have performed numerical simulations showing the sensitivity of the resulting pattern on the alignment of the gratings, which limits the resolution of the method in the present case. We believe that further reduction of the feature size should be possible with this method by either improving the mask fabrication strategy for smaller period gratings and more precision in the positioning of the gratings or by employing the second order diffraction in EUV-IL. ${ }^{14}$ The presented method has a further advantage of being achromatic, i.e., it does not need temporal coherence of the EUV light source because of the diffraction-based interference. Therefore, we can also fully take advantage of this high-efficiency achromatic method, and easily spread this method to most available EUV sources, specifically, tabletop EUV sources. Since HSQ has a refractive index of about 1.4 in the visible wavelength range, it provides a reasonable contrast to air and therefore the generated patterns can be used directly as photonic devices. Moreover, due to its high etch resistance, HSQ is a suitable etching mask for transferring its pattern into other highcontrast photonic, magnetic, and semiconductor materials. Therefore, the demonstrated kagome lattice structures can be used in various applications such as in the study of frustrated physical systems, nanophotonics, and nanoplasmonics. In particular, fabrication of frustrated magnetic nanostructures over large areas will simplify their characterization with synchrotron sources and neutron scattering experiments.

The authors thank Michaela Vockenhuber for technical support. This project was funded by the Nano-Argovia program of Swiss Nanoscience Institute Basel (SNI). Part of this work was performed at the Swiss Light Source (SLS), Paul Scherrer Institute, 5232 Villigen PSI, Switzerland. 
${ }^{1}$ I. Syozi, Prog. Theor. Phys. 6, 306 (1951).

${ }^{2}$ M. Mekata, Phys. Today 56(2), 12 (2003).

${ }^{3}$ A. Mielke, J. Phys. A 24, L73 (1991).

${ }^{4}$ P. Mohan, J. Motohisa, and T. Fukui, Appl. Phys. Lett. 84, 2664 (2004).

${ }^{5}$ M. L. Neel, Ann. Geophys. 5, 99 (1949); W. F. Brown, Phys. Rev. 130, 1677 (1963).

${ }^{6}$ E. Mengotti, L. J. Heyderman, A. F. Rodriguez, F. Nolting, R. V. Hügli, and H. B. Braun, Nature Phys. 7, 68 (2011).

${ }^{7}$ M. Boguslawski, P. Rose, and C. Denz, Appl. Phys. Lett. 98, 06111 (2011).

${ }^{8}$ P. Rose, M. Boguslawski, and C. Denz, New J. Phys. 14, 033018 (2012).

${ }^{9}$ C. Wagner and N. Harned, Nat. Photonics 4, 24 (2010).
${ }^{10}$ V. Auzelyte, C. Dais, P. Farquet, D. Grützmacher, L. H. Heyderman, F. Luo, S. Olliges, C. Padeste, P. K. Sahoo, T. Thomson, A. Turchanin, C. David, and H. H. Solak, J. Micro/Nanolith. MEMS MOEMS 8, 021204 (2009).

${ }^{11}$ B. Terhalle, A. Langner, B. Päivanranta, and Y. Ekinci, Proc. SPIE. 8102, 81020V-1 (2011).

${ }^{12}$ A. Langner, B. Päivanranta, B. Terhalle, and Y. Ekinci, Nanotechnology 23, 105303 (2012).

${ }^{13}$ L. Wang, B. Terhalle, M. Hojeij, V. A. Guzenko, and Y. Ekinci, J. Vac. Sci. Technol. 30, 031603 (2012).

${ }^{14}$ B. Päivanranta, A. Langner, E. Kirk, C. David, and Y. Ekinci, Nanotechnology 22, 375302 (2011). 